Int. J. Dev. Biol. 59: 461-464 (2015)

doi: $10.1387 / \mathrm{ijdb} .150280 \mathrm{dr}$

\title{
Angiogenesis and hyperbaric oxygen in the chick embryo chorioallantoic membrane
}

\author{
UMBERTO MONTECORBOLI ${ }^{1,2}$, TIZIANA ANNESE ${ }^{2}$, CHRISTIAN MARINACCIO ${ }^{2}$, and DOMENICO RIBATTI ${ }^{*, 2,3}$ \\ ${ }^{1}$ Unit of Hyperbaric Medicine, Ospedale "San Paolo", ${ }^{2}$ Department of Basic Medical Sciences, Neurosciences, and \\ Sensory Organs, University of Bari Medical School and ${ }^{3}$ National Cancer Institute "Giovanni Paolo II", Bari, Italy
}

\begin{abstract}
Hyperbaric Oxygen Therapy (HBOT) is increasingly applied in different areas of medical practice. The oxy-hyperbarism effects are not well understood in cancer malignancy. One unique feature of cancer is the presence of hypoxic regions that are insensitive to conventional therapies. It is possible to alter the hypoxic state and produce reactive oxygen species for better treatment outcome by HBOT. In the present study, we determined the effects of HBOT on angiogenesis, a signature of cancer progression, by using the chick chorioallantoic membrane (CAM) in vivo assy. CAMs were exposed to 2.0 ATA (atmospheres absolute) for $30 \mathrm{~min}$ of hyperbaric oxygen on the $\mathbf{6}^{\text {th }}$ and $7^{\text {th }}$ days of incubation (ED6, ED7). On the $10-11^{\text {th }}$ day of incubation, CAMs were excised from eggs, fixed and analysed using APERIO ImageScope software. HBOT outcomes were evaluated quantifying the volumetric area occupied by blood vessels and calculating the number of blood vessel ramifications. Results indicated that CAMs treated at ED6 and ED7 had a significantly higher CAM vascularization and an increased number of blood vessel ramifications (+82\% higher for ED6) compared to untreated CAMs (ED6 $=63.3 \pm 2.5$ and $E D 7=57.7 \pm 5.5$ vs. $C T R L=34.7 \pm 2.5$ ). Thus, HBOT induces an angiogenic response in treated CAMs through a classic sprouting mechanism.
\end{abstract}

KEY WORDS: angiogenesis, chorioallantoic membrane, hyperbaric oxygen

The variations of oxygen tissue tensions and the various conditions related to normoxia, hypoxia and hyperoxia are determining factors on the metabolic pathways and the formation of new blood vessels in several pathological conditions. The Hyperbaric Oxygen Therapy (HBOT) is a treatment used primarily to improve or cure disorders related to hypoxia and ischemia which, by increasing the amount and the partial tension of the oxygen (PpO2) dissolved in the plasma, enhances its diffusion in the tissues.

The major international scientific societies defined several clinical indications for which the therapeutic use Oxygen Hyperbaric was approved including Gas Embolism Hypertension (EGA), Decompression Illness (DCI), Carbon Monoxide poisoning, acute traumatic ischemia and chronic wounds (Gesell, 2008; Mathieu, 2005).

Since its introduction in clinical practice, studies conducted on HBOT and cancer have been mainly focused on the stimulating role that pressurized oxygen may have on cancer growth. Systematic reviews of HBOT and cancer have described the use of HBOT in patients with malignancy (Daruwalla and Christophi, 2006; Hoggan and Cameron, 2014; Mayer et al., 2005; Moen and Stuhr, 2012). However, several unsolved questions remain connected with HBOT treatment, including severe DNA damage and genetic instability leading to reactivation or augmentation of existing cancer, development of fragile and porous capillaries and associated higher chance of metastasis, initiating aneuplody causing delay in DNA repair of surrounding normal or stromal cells.

The effects of the mechanisms action of hypoxia are extensively investigated in clinical and experimental settings, but less known are the effects that the conditions of hyperoxia and mostly the one of oxy-hyperbarism may have in biological processes.

The chick embryo chorioallantoic membrane (CAM) is an extraembryonic membrane which serves as a gas exchange surface and its function is supported by a dense capillary network. Because of its extensive vascularization and easy accessibility, the CAM has been broadly used to study the morphofunctional aspects of the angiogenesis process in vivo and to investigate the efficacy and mechanisms of action of pro-angiogenic and anti-angiogenic

Abbreviations used in this paper: CAM, chick embryo chorioallantoic membrane; HBOT, hyperbaric oxygen therapy.

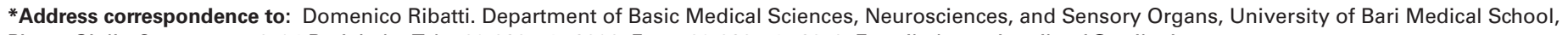
Piazza Giulio Cesare, 11, 70124 Bari, Italy. Tel: +39-080-547-8326. Fax: +39-080-547-8310. E-mail: domenico.ribatti@uniba.it
}

Accepted: 15 October 2015.

ISSN: Online 1696-3547, Print 0214-6282 

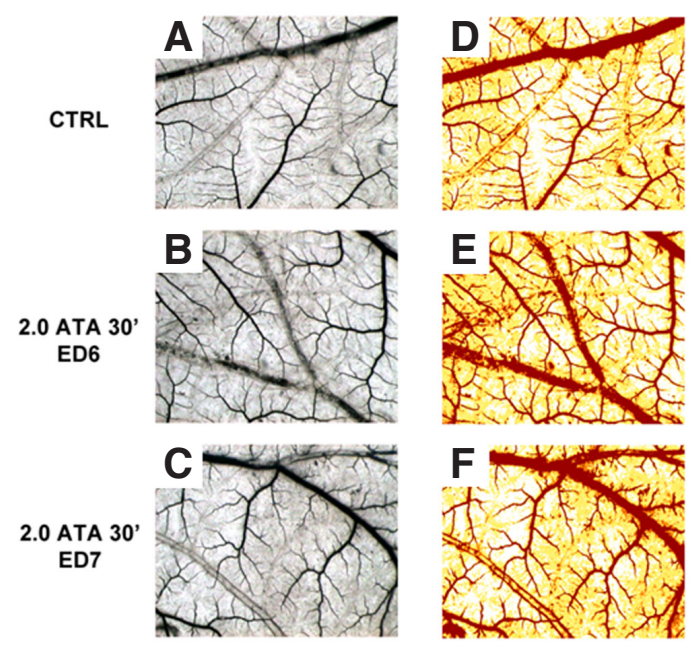

Fig. 1. Differences in vascularization between control, ED6 and ED7. Chicken eggs were treated with 2.0 ATA for a 30-min exposure of Hyperbaric Oxygen on the $6^{\text {th }}$ (ED6) and $7^{\text {th }}$ (ED7) day of incubation respectively, while untreated eggs were used as controls (CTRL). On the 10-11 th day of incubation, CAMs were excised from eggs, fixed and examined. Stereomicroscope images were converted in gray-scale (A-C) and analyzed using the Aperio Positive Pixel Count algorithm; see mark-up images (D-F). CAMs treated at ED6 and ED7 show a significant higher percentage of strong positive pixels than control ones ( $E D 6=29.76 \pm 3.05$ and $E D 7=30.58 \pm 2.94 \mathrm{vs} C T R L=23.98 \pm 1.75)$. Mark-up image color code: Red, strong positive pixels; Orange, medium positive pixels; Yellow, weak positive pixels. ${ }^{*} p<0.05$.

natural and synthetic molecules (Ribatti, 2008).

The aim of this study is to evaluate the possible effects that exposure to Hyperbaric Oxygen (HBO) on the process of angiogenesis by using as experimental in vivo model the chick embryo CAM.

\section{Results and Discussion}

Image analysis shows significant differences between control CAM's and treated CAMs. The percentage of strong positive pixels which identifies blood vessels in the stereomicroscopic images shows a significant difference between treated and control CAMs. Specifically, CAMs treated at ED7 showed a higher percentage of strong positive pixels (mean $=30.58 ; \mathrm{SD}=2.939$ ) when compared with control CAMs (mean $=23.98, S D=1.750$ ). A higher percentage of strong positive pixels was found in CAMs treated at ED6 ( mean $=29.76$; SD $=3.051$ ) when compared with control CAMs (mean $=23.98, S D=1.750)$, as well. No statistical significance was found between the two treated groups (Fig. 1).

Counting of primary, secondary and tertiary blood vessel ramifications in a magnified particular stereomicroscopic of all the images revealed significant differences between the experimental groups. In particular, the number of blood vessel ramifications were higher in CAMs treated at ED7 (mean $=57.67, \mathrm{SD}=5.508$ ) when

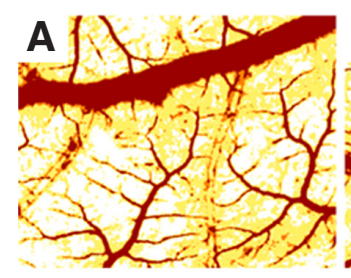

CTRL

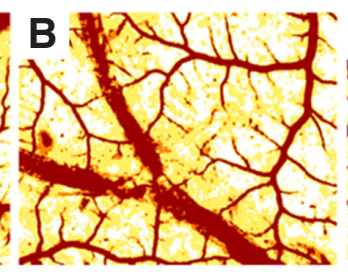

2.0 ATA 30' ED6

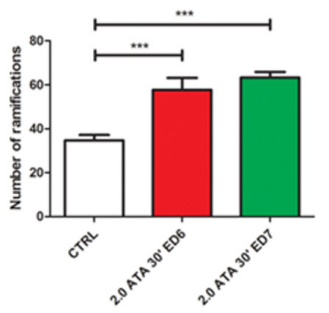

compared with control CAMs (mean $=34.67, \mathrm{SD}=2.517$ ). CAMs treated at ED6 also showed a higher number of blood vessel ramifications (mean $=63.33, \mathrm{SD}=2.517$ ) when compared with control CAMs (mean $=34.67, \mathrm{SD}=2.517$ ). No statistical significance was found between the two treated groups (Fig. 2). The presence of an higher number of blood vessel ramifications in CAMs treated at ED7 when compared with control CAMs was confirmed also by histological analysis (Fig. 3).

Vascular remodeling is a fundamental physiological process during development and acclimatization to adverse environments, and a multitude of factors are involved in the angiogenic response during vascular remodeling. Oxygen tension is positively correlated with collagen production, bacterial killing, epithelialization, and angiogenesis (Knighton, 1990).

The results of this study have demonstrated that $\mathrm{HBO}$ is able to induce an angiogenic response in the treated CAMs as compared to control ones, and are in accord with a general effect of high tension of oxygen of enhancement of growth of chick embryo, as previously demonstrated by Stock and co-workers (Stock et al., 1983).

The principal mechanisms of action of $\mathrm{HBO}$ are based on the intracellular production of Reactive Oxygen Species (ROS) including superoxide anions (O2-), hydroxyl radicals $\left(\mathrm{OH}^{-}\right)$, and hydrogen peroxide $\left(\mathrm{H}_{2} \mathrm{O}_{2}\right)$. Increased release of ROS generated by cyclooxygenase- 1 in endothelial cells might produce vasoconstriction by inhibiting the synthesis or the action of vasodilatory components such as prostacyclin or nitric oxide (Stuart et al., 1984). Moreover, this latter is a strong modulator of the angiogenesis (Aicher et al., 2003). At micromolar concentrations, $\mathrm{H}_{2} \mathrm{O}_{2}$ induces vascular endothelial

Fig. 2. Blood vessel ramifications counting between control, ED6 and ED7. Chicken eggs were treated with 2.0 ATA for a 30-min exposure to hyperbaric oxygen on the $6^{\text {th }}$ (ED6) and $7^{\text {th }}$ (ED7) day of incubation respectively, while untreated eggs were used as controls (CTRL). On the 10-11 th day of incubation, CAMs were excised from eggs, fixed and examined. Mark-up images (A-C) analyzed using the Aperio Positive Pixel Count algorithm were used to count primary, secondaryand tertiary blood vessels ramifications. CAMs treated at ED6 and ED7 showed a significant increase in the number of blood vessel ramifications compared to control ones $(E D 6=63.33 \pm 2.52$ and $E D 7=57.67 \pm 5.51$ vs $C T R L=34.67 \pm 2.52$ ). ${ }^{* *} p<0.001$. 

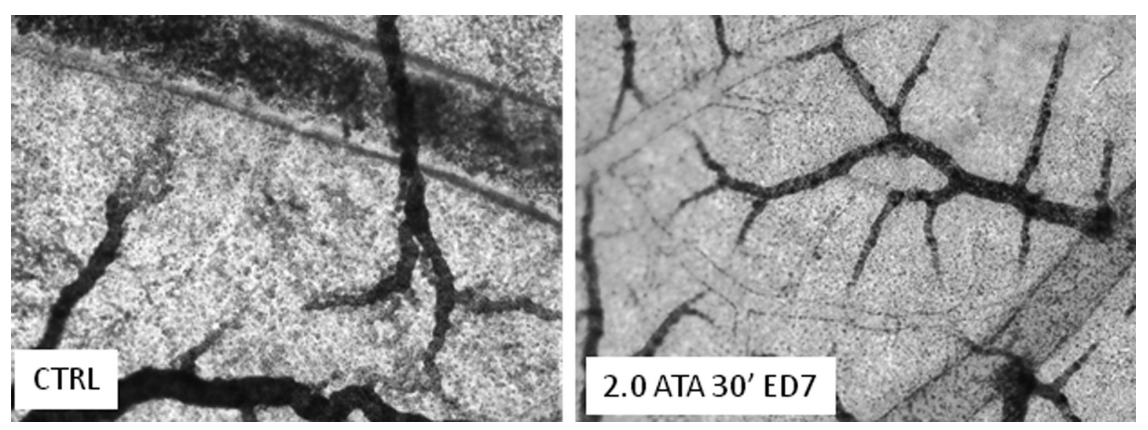

Fig. 3. Microscopic features of vascular tree in control CAM (CTRL) and CAM treated at ED 7 (2.0 ATA 30' ED7). Note an higher number of vascular ramifications in the experimental condition. Original magnification, $5 x$. cell growth factor (VEGF) expression (Sen, 2002).

On the contrary, oxidative stress caused by high oxygen exposure suppress expression of angiogenic factors such as VEGF (Thébaud and Abman, 2007). Moreover, hyperoxia reduces the number of circulating endothelial cell precursors in blood and lung of newborn mice (Balasubramaniam etal., 2007), induces apoptosis of endothelial cells (Gu et al., 2003), and inhibits endothelial cell proliferation (D'Amore and Sweet, 1987).

As concerns the CAM vascularization, primitive vessels proliferate and differentiate into an arterio-venous system until day 8 , thus originating a network of capillaries that migrate to occupy an area beneath the chorion and mediate gas exchanges with the outer environment. Rapid capillary proliferation goes until ED11; thereafter, their mitotic index declines just as rapidly, and the vascular system attains its final arrangement on ED18, just before hatching (Ausprunk et al., 1974). A morphometric investigation by DeFouw et al., has shown rapid extension of the CAM surface between ED6 and ED14, and during this period, the number of feed vessels increased predominantly due to growth and remodeling after ED10 (DeFouw et al., 1989). According to Schlatter et al., CAM vascularization undergoes three phases of development with both sprouting and intussusceptive microvascular growth: in the early phase (days 5-7) multiple capillary sprouts invade the mesenchyme, fuse and form the primary capillary plexus. During the second (intermediate phase) (days 8-12), sprouts are no longer present since they have been replaced by tissue pillars expression of intussusceptive microvascular growth, with a maximal frequency at day 11 (Schlatter et al., 1997). During the late phase (day 13 and older), the growing pillars increase in size to from inter-capillary meshes more than $2.5 \mu \mathrm{m}$ in diameter.

Development of the response to hypoxia is better described than that to hyperoxia. Dusseau and Hutchins studied the effects of hypoxia on the number of pre and post-capillary vessels in the CAM and demonstrated that the low oxygen regimen stimulated a preferential increase in the number of arterioles (Dusseau and Hutchins, 1989). In our experimental condition, HBO induces an angiogenic response through a classic sprouting mechanism, because the time of treatment correspond to early phase of CAM vascular growth, in which angiogenic occurs. This is also confirmed by an increase in the number of blood vessel ramifications in experimental group, as compared to control ones. However, also in this case, in contrast with our study, other Authors have previously studied the effects of hyperoxia on the angiogenesis process in the CAM. Chick eggs were incubated in various oxygen atmospheres beginning on the 7th day of development, and vascularity was measured on the 14th day. Measurements included vessel endpoint density, length density, fractional image area, and a vascular density index, using a computerized image analysis system, and the results demonstrated that hyperoxia inhibits angiogenesis (Strick et al., 1991).

\section{Materials and Methods}

Two hundred fertilized White Leghorn chicken eggs were incubated at $37^{\circ} \mathrm{C}$ at constant humidity (60\%). On day 3 of incubation, a square window was opened in the egg shell, and 2 to $3 \mathrm{ml}$ of albumen was removed to allow detachment of the developing CAM from the shell. The window was sealed with a glass, and the eggs returned to the incubator. Embryo manipulation did not alter its development. The possibility to look inside the embryo through the window allows to verify the vitality of the embryo. For each batch of chicken eggs supplied, three randomized groups were set up: treatment with Hyperbaric Oxygen on the $6^{\text {th }}$ day of incubation (ED6), treatment with $\mathrm{HBO}$ at $7^{\text {th }}$ day of incubation (ED7) and a control group (CTRL).

For security reasons the system embryo/bag saturated in oxygen was then placed in a small recompression chamber for experimental use (GlobalMed Pontenure srl-Italy) and pressurized air at a pressure of 2.0 ATA (atmospheres absolute) for a 30-min exposure of HBO. After treatment, the embryos were removed from the bag and placed back in an incubator. After four days of post-treatment incubation, CAMs were excised and then fixed with Karnovsky's fixative for $2 \mathrm{~h}$ at room temperature. CAMs were then examined and photographed with a stereomicroscope equipped with a camera (Olympus Italia, Rozzano, Italy) at $10 \mathrm{X}$ magnification. Finally, CAMs were processed for light microscopy. CAM portions were removed and embedded in paraffin. Fifteen $u$ m sections were cut according to a plane parallel to the surface of the CAM, stained with $0.5 \%$ aqueous solution of toluidine blue (Merck Biochemica), and observed under a Leitz-Dialux 20 light microscope (Leitz, Wetzlar, Germany).

\section{Image analysis}

Microscopic images obtained from the stereomicroscope were converted into gray-scale and analyzed using the Aperio Positive Pixel Count algorithm embedded in the ImageScope v.11.2.0.780 (Leica Biosystems Nussloch, Germany) as previously described. Briefly, the algorithm input parameters were initially set to obtain the identification of pixels related to the blood vessels as strong and positive as to the background medium and weak positive and tuned to minimize non-specific pixel recognition as strong positive. The ratio between the number of strong positive pixels and the sum of weak, medium and strong positive pixels is the morphometric value used to quantitate CAM vascularization across the experimental groups.

In additions, random areas of the images were magnified and the primary, secondary and tertiary blood vessel ramifications were counted in all the experimental groups. All the analysis were performed on images with equal area.

\section{Statistical analysis}

Fold change data are reported as means \pm SD. The Graph Pad Prim 5.0 statistical package (GraphPad Software, San Diego, CA, USA) was 
used for the analysis and $\mathrm{P}<0.05$ was considered as the limit for statistical significance.

\section{References}

AICHER, A., HEESCHEN, C., MILDNER-RIHM, C., URBICH, C., IHLING, C., TECHNAU-IHLING, K., ZEIHER, A.M. and DIMMELER, S. (2003). Essential role of endothelial nitric oxide synthase for mobilization of stem and progenitor cells. Nature Med. 9: 1370-1376.

AUSPRUNK, D.H., KNIGHTON, D.R. and FOLKMAN, J. (1974). Differentiation of vascular endothelium in the chick chorioallantois: Astructural and autoradiographic study. Dev. Biol. 38: 237-248.

BALASUBRAMANIAM, V., MERVIS, C.F., MAXEY,A.M., MARKHAM, N.E. andABMAN, S.H. (2007). Hyperoxia reduces bone marrow, circulating, and lung endothelial progenitor cells in the developing lung: implications for the pathogenesis of bronchopulmonary dysplasia. AJP: Lung Cell. Molec. Physiol. 292: L1073-L1084.

D'AMORE, P.A. and SWEET, E. (1987). Effects of hyperoxia on microvascular cells in vitro. In vitro Cell. Dev. Biol. 23: 123-128.

DARUWALLA, J. and CHRISTOPHI, C. (2006). Hyperbaric Oxygen Therapy for Malignancy: A Review. World J. Surg. 30: 2112-2131.

DEFOUW, D.O., RIZZO, V.J., STEINFELD, R. and FEINBERG, R.N. (1989). Mapping of the microcirculation in the chick chorioallantoic membrane during normal angiogenesis. Microvascular Res. 38: 136-147.

DUSSEAU, J.W. and HUTCHINS, P.M. (1989). Microvascular responses to chronic hypoxia by the chick chorioallantoic membrane: A morphometric analysis. Microvascular Res. 37: 138-147.

GESELL, L. (2008). Hyperbaric Oxygen Therapy: Indications, 12th Edition. The Hyperbaric Oxygen therapy Committee Report. Durham, NC, Undersea and Hyperbaric Medical Society.

GU, X., EL-REMESSY, A.B., BROOKS, S.E., AL-SHABRAWEY, M., TSAI, N.T. and CALDWELL, R.B. (2003). Hyperoxia induces retinal vascular endothelial cell apoptosis through formation of peroxynitrite. AJP: Cell Physiol. 285: C546-C554.
HOGGAN, B.L. and CAMERON, A.L. (2014). Systematic review of hyperbaric oxygen therapy for the treatment of non-neurological soft tissue radiation-related injuries. Support Care Cancer 22: 1715-1726.

KNIGHTON, D.R. (1990). Oxygen as an Antibiotic. Arch Surg 125: 97.

MATHIEU. (2005). 7th European Consensus Conference on Hyperbaric Medicine: Lille, 3rd - 4th December 2004. Europ J Underwater Hyperbaric Med 6: 29-38.

MAYER, R., HAMILTON-FARRELL, M.R., VAN DER KLEIJ, A.J., SCHMUTZ, J., GRANSTRÖM, G., SICKO, Z., MELAMED, Y., CARL, U.M., HARTMANN, K.A. JANSEN, E.C. et al., (2005). Hyperbaric Oxygen and Radiotherapy. Strahlenther Onkol 181: 113-123.

MOEN, I. and STUHR, L.E.B. (2012). Hyperbaric oxygen therapy and cancer-a review. Targeted Oncoll. 7: 233-242.

RIBATTI, D. (2008). Chapter 5 Chick Embryo Chorioallantoic Membrane as a Useful Tool to Study Angiogenesis. In Int. Rev. Cell. Molec. Biol., (ed., pp. 181-224: Elsevier BV.

SCHLATTER, P., KÖNIG, M.F., KARLSSON, L.M. and BURRI, P.H. (1997). Quantitative Study of Intussusceptive Capillary Growth in the Chorioallantoic Membrane (CAM) of the Chicken Embryo. Microvascular Res. 54: 65-73.

SEN, C.K. (2002). Oxidant-induced Vascular Endothelial Growth Factor Expression in Human Keratinocytes and Cutaneous Wound Healing. J. Biol. Chem. 277: 33284-33290.

STOCK, M.K., FRANCISCO, D.L. and METCALFE, J. (1983). Organ growth in chick embryos incubated in $40 \%$ or $70 \%$ oxygen. Respiration Physiol. 52: 1-11.

STRICK, D.M., WAYCASTER, R.L., MONTANI, J.P., GAY, W.J. and ADAIR, T.H. (1991). Morphometric measurements of chorioallantoic membrane vascularity: effects of hypoxia and hyperoxia. Am J Physiol 260: $\mathrm{H} 1385-\mathrm{H} 1389$.

STUART, M.J., SETTY, Y., WALENGA, R.W., GRAEBER, J.E. and GANLEY, C. (1984). Effects of hyperoxia and hypoxia on vascular prostacyclin formation in vitro. Pediatrics 74: 548-553.

THÉBAUD, B. and ABMAN, S.H. (2007). Bronchopulmonary Dysplasia. Am J Respir Crit Care Med 175: 978-985. 


\section{Further Related Reading, published previously in the Int. J. Dev. Biol.}

Epithelial-mesenchymal interactions: a fundamental Developmental Biology mechanism Domenico Ribatti and Marcello Santoiemma

Int. J. Dev. Biol. (2014) 58: 303-306

http://dx.doi.org/10.1387/ijdb.140143dr

Differential expression of angiogenic and anti-angiogenic molecules in the chick embryo chorioallantoic membrane and selected organs during embryonic development

Christian Marinaccio, Beatrice Nico and Domenico Ribatti

Int. J. Dev. Biol. (2013) 57: 907-916

http://dx.doi.org/10.1387/ijdb.130317dr

Hypoxia-inducible factor 1 controls the expression of the uncoordinated-5-B receptor, but not of Netrin-1, in first trimester human placenta

Mbarka Dakouane-Giudicelli, Nadia Alfaidy, Perrine Bayle, Alexandre Tassin de Nonneville, Vivien Studer, Patrick Rozenberg and Philippe de Mazancourt

Int. J. Dev. Biol. (2011) 55: 981-987

http://dx.doi.org/10.1387/ijdb.103276md

Zebrafish enhancer trap line recapitulates embryonic aquaporin 1a expression pattern in vascular endothelial cells

Kira Rehn, Kuan Shen Wong, Darius Balciunas and Saulius Sumanas

Int. J. Dev. Biol. (2011) 55: 613-618

http://dx.doi.org/10.1387/ijdb.103249kp

Zebrafish embryo, a tool to study tumor angiogenesis

Chiara Tobia, Giulia De Sena and Marco Presta

Int. J. Dev. Biol. (2011) 55: 505-509

http://dx.doi.org/10.1387/ijdb.103238ct

Role of laminins in physiological and pathological angiogenesis

Patricia Simon-Assmann, Gertraud Orend, Elmina Mammadova-Bach, Caroline Spenlé and Olivier Lefebvre

Int. J. Dev. Biol. (2011) 55: 455-465

http://dx.doi.org/10.1387/ijdb.103223ps

\section{A brief history of angiogenesis assays}

Anca-Maria Cimpean, Domenico Ribatti and Marius Raica

Int. J. Dev. Biol. (2011) 55: 377-382

5 yr ISI Impact Factor $(2013)=2.879$
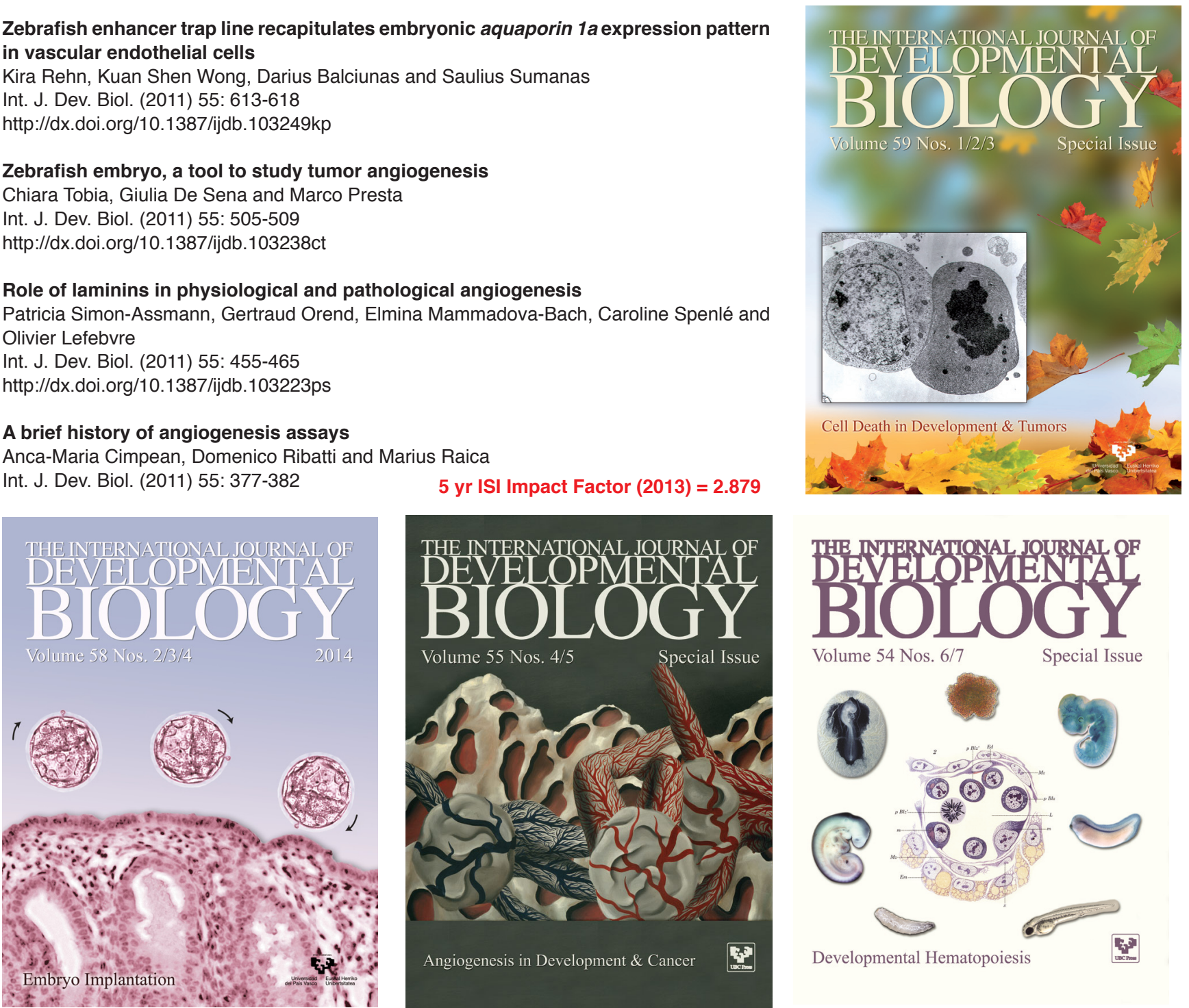

Volume 54 Nos. 6/7

Special Issue

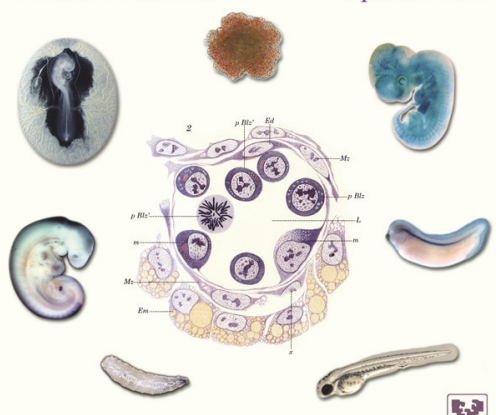

Developmental Hematopoiesis

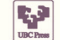

